\title{
Analytical Spatial Resolution in EPMA: What is it and How can it be Estimated?
}

\author{
Aurélien Moy ${ }^{1}$ and John Fournelle ${ }^{1}$
}

${ }^{1}$ Department of Geoscience, University of Wisconsin, Madison, WI 53706, USA.

The imaging benefits of field emission SEM are clear: edge resolution down to ten or so nanometers, particularly for secondary electron images. Edge resolution is defined as the ability to distinguish two adjacent features, based upon difference in signal intensity (e.g. 88\% - 12\%). However, improving EPMA analytical spatial resolution is complex. Changing the electron source on an electron microprobe from tungsten to a field emission one, and assuming an immediate improvement in analytical resolution with the typical accelerating voltage $(15 \mathrm{kV})$, is problematic. Landing beam diameter is one factor, but most critical is the immediate electron scattering once electrons enter the target. Analytical resolution, then, is critically related to the energy of the incident electrons. Monte Carlo electron-target modeling demonstrates the necessity of decreasing beam energy (Figure 1).

Reducing the accelerating voltage, with its benefit of constraining the incident electrons to a tighter interaction volume, however, leads to further complications in many cases - the necessity to alter the characteristic X-rays lines being used if the 'normal' ones are not being excited. This is particularly the case for the transition metals (e.g. Fe) and is discussed in an associated presentation at this conference [1]. And operation at lower $\mathrm{keV}(5-10 \mathrm{keV})$ accentuates the significance of the upper region of the sample and issues of carbon contamination and oxide coats on metals, which can impact the quality of the EPMA results. Additionally, the combination of tight FE beam diameter and reduced interaction volume may lead to alteration/damage of the target material.

Working with EPMA of sub-micron phases, one inevitably asks "How small an object can you analyze?" Traditionally, equations for "X-ray range" and more recently, Monte Carlo simulations, have been used for estimates. However, it is useful to quote Barkshire et al [2]: "... instead of simply being able to resolve the feature in an image, the entire X-ray information volume must be contained within the feature. Thus, for this purpose a more reasonable definition of spatial resolution is the diameter of a feature such that it will include some fraction of the total X-ray intensity, e.g. $99 \%$." This definition for lateral analytical spatial resolution is the one we believe should be used in EPMA.

We found NBS/NIST "failed standard" K409 to be useful for evaluating the various equations and Monte Carlo simulations; this material is a silicate glass with $\mathrm{Na}, \mathrm{Al}$ and Fe present [3]. Rather than being a homogenous material, it has abundant $\sim 800 \mathrm{~nm}$ magnetite $\left(\mathrm{Fe}_{3} \mathrm{O}_{4}\right)$ crystals. There are different spacings of glass between the crystals, so FE-EPMA measurements of the glass at 5 and $7 \mathrm{keV}$ were performed, and the results compared with the traditional X-ray range and Monte Carlo simulation results. The results suggested there was little if any improvement in decreasing the accelerating voltage from 7 to $5 \mathrm{kV}$.

However, the geometry of this sample is not optimal for the usual situation of evaluating small equant to semi-equant inclusions within some other matrix. For this more common situation, we have recently developed an application to estimate particle/inclusion lateral analytical spatial resolution, using a simple graphical interface to the powerful PENEPMA Monte Carlo program, WISC-Resolution. Particle and matrix compositions are defined by the user; the particle geometry can be spherical or rectangular. As many parallel simulations can be run simultaneously as there are processors on the computer. 
Simulations can take some time (hours) to reach desired tight precision (e.g. 1\%) but immediate output is shown (i.e., wider error bars) which can be useful.

We have tested the program on one published example [4], small $\mathrm{Cu}-\mathrm{Mg}-\mathrm{Pd}$ precipitates in a $\mathrm{Mg}-\mathrm{Pd}$ matrix at $6 \mathrm{keV}$. For this sample, the calculated analytical resolution, in radius, for the element $\mathrm{Mg}(\mathrm{K} \alpha$ line; $1.254 \mathrm{keV}), \mathrm{Pd}(\mathrm{L} \alpha$ line; $2.838 \mathrm{keV}$ ) and $\mathrm{Cu}$ (L $\alpha$ line; $0.928 \mathrm{keV}$ ) are $\sim 0.35-.4 \mu \mathrm{m}, \sim 0.35-.4 \mu \mathrm{m}$ and $\sim 0.35-.4 \mu \mathrm{m}$, respectively. After scaling, the simulated results match well the experimental data (Figures 2). WISC-Resolution software is available to interested parties.

\section{References:}

[1] A Moy and J Fournelle, Microscopy and Microanalysis (this conference).

[2] I Barkshire et al, Microchimica Acta 132 (2000), p. 113.

[3] J Fournelle et al, IOP Conf. Series: Materials Science and Engineering 109 (2016).

[4] C Hombourger and M Outrequin, Microscopy Today 21(03) (2013), p. 10.

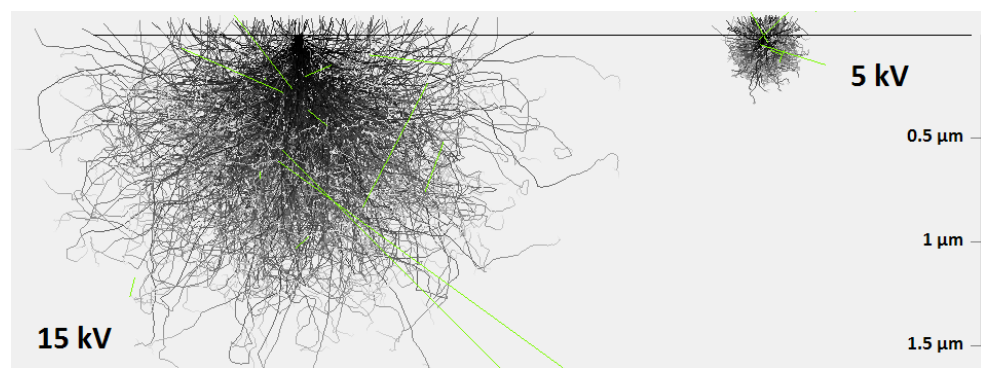

Figure 1. PENELOPE Monte Carlo simulations of K409 glass at 15 and $5 \mathrm{kV}$ showing extent of electron scatter in sample.

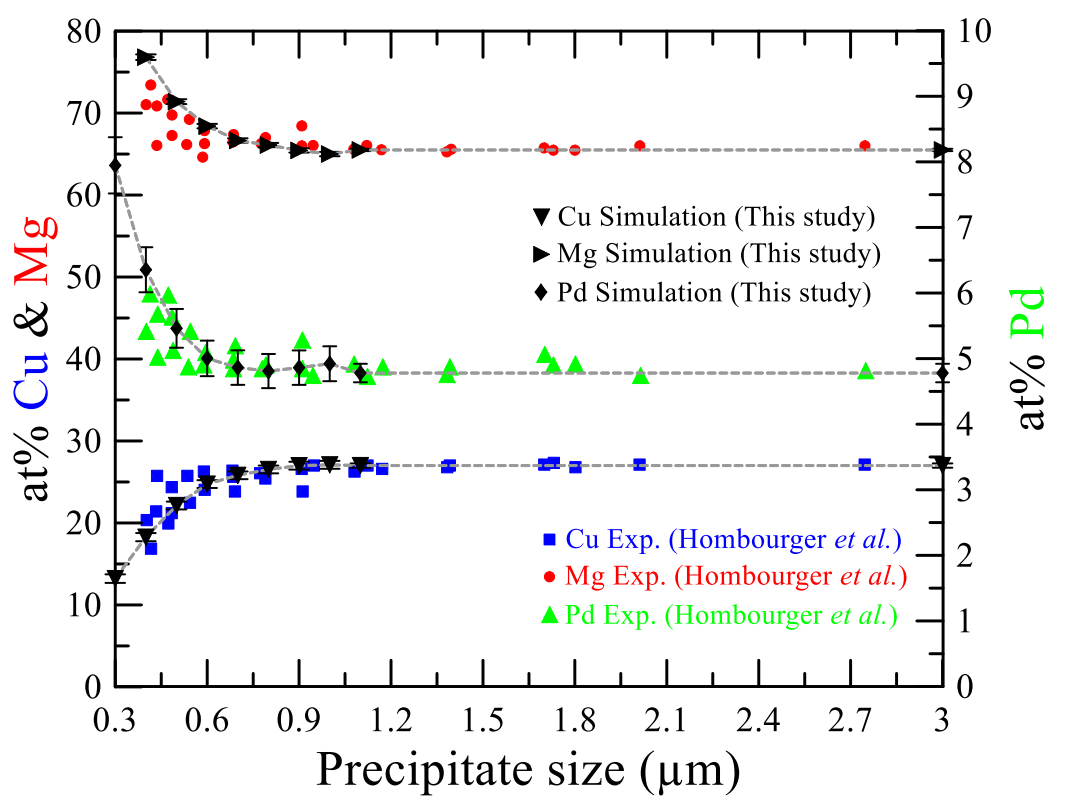

Figure 2. WISC-Resolution output for hemispherical $\mathrm{Cu}-\mathrm{Mg}$-Pd precipitates in $\mathrm{Mg}$-Pd. The scaled PENEPMA simulation, performed at $6 \mathrm{kV}$, matches well the experimental data of Hombourger and Outrequin [4]. 